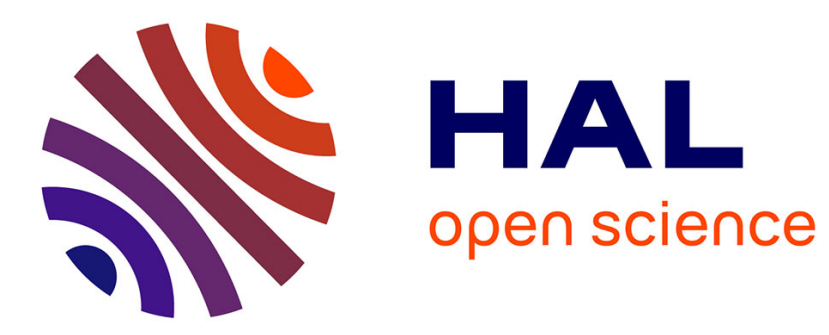

\title{
Game-Theoretic based Power Allocation for a Full Duplex D2D Network
}

\author{
Hussein Chour, Youssef Nasser, Faouzi Bader, Oussama Bazzi
}

\section{To cite this version:}

Hussein Chour, Youssef Nasser, Faouzi Bader, Oussama Bazzi. Game-Theoretic based Power Allocation for a Full Duplex D2D Network. IEEE International Workshop on Computer Aided Modeling and Design of Communication Links and Networks (CAMAD), Sep 2019, Limassol, Cyprus. 10.1109/CAMAD.2019.8858466 . hal-02218116

\section{HAL Id: hal-02218116 https://hal.science/hal-02218116}

Submitted on 31 Jul 2019

HAL is a multi-disciplinary open access archive for the deposit and dissemination of scientific research documents, whether they are published or not. The documents may come from teaching and research institutions in France or abroad, or from public or private research centers.
L'archive ouverte pluridisciplinaire HAL, est destinée au dépôt et à la diffusion de documents scientifiques de niveau recherche, publiés ou non, émanant des établissements d'enseignement et de recherche français ou étrangers, des laboratoires publics ou privés. 


\title{
Game-Theoretic based Power Allocation for a Full Duplex D2D Network
}

\author{
Hussein CHOUR ${ }^{1,2}$, Youssef NASSER ${ }^{3}$, Faouzi BADER ${ }^{1}$, and Oussama BAZZI ${ }^{2}$ \\ ${ }^{1}$ CentraleSupélec/IETR, CentraleSupélec Campus de Rennes, 35510 Cesson-Sévigné, France \\ ${ }^{2}$ Physics and Electronics Department, Faculty of Science 1, Lebanese University, Beirut, Lebanon \\ ${ }^{3}$ ECE Department, American University of Beirut, Bliss Street, Beirut, Lebanon, \\ Email:\{hussein.chour@supelec.fr, youssef.nasser@ieee.org, faouzi.bader@supelec.fr, obazzi@ul.edu.lb\}
}

\begin{abstract}
This paper tackles the power allocation optimization problem of a Full duplex(FD) D2D underlaying cellular network. In particular, we aim at providing a distributed power allocation algorithm for this type of network. Towards this end, first, we formulate the PA problem as a non-cooperative game in which each user decides how much power to transmit over its allocated channel to maximize its link's energy-efficiency (EE). Next, we show that this game admits a unique Nash equilibrium (NE) point which can be obtained through an iterative process. After that, we show that this iterative algorithm can be implemented in a fully distributed manner. Finally, we compare our proposed distributed algorithm with the conventional centralized algorithms and simulation results show the importance of the proposed solution. Index Terms-full-duplex transmission, device-to-device (D2D) communication, power allocation, non-cooperative game
\end{abstract}

\section{INTRODUCTION}

With the scarcity of the cellular radio spectrum and the booming of the users' data traffic demands [1], the need for innovative technologies that can fulfill the users' requirement and use the cellular radio band efficiently has become a substantial part in the coming radio network $(5 \mathrm{G})$. In this respect, Full Duplex (FD) transmission and device-to-device (D2D) communication are proposed to increase the efficiency of the cellular spectrum and enhance the cellular network users' experience. D2D allows direct communication between nearby devices without passing the base station (BS) [2]. Further, FD enables a device to transmit and receive signals on the same carrier at the same time [3].

Since D2D communication happens between devices located within a short distance of each other, merging D2D Communication and FD technology can further improve the cellular users' throughput and the utilization of the cellular radio spectrum [4]. However, the actual FD devices introduce new challenges on the D2D network. For example, the current FD devices cannot totally eliminate the self-interference (SI) imposed by the transmitter on the receiver within the same FD transceiver [3]. Hence, the performance of FD communication is highly affected by the residual self-interference (RSI) power which in turn is tightly related to the transmitter power value and the applied SI cancellation technique at the FD device. In addition, using the FD technique creates additional interference which may overwhelm the conventional cellular link. Thus, proper power allocation is needed to exploit the benefits of FD transmission in a D2D network and guarantee the quality of service (QoS) of the users. An overview of the related power allocation schemes in an FD-D2D network is given below.

\section{A. Related works}

The work in [5] addressed the power optimization problem of a single full-duplex D2D pair underlaying cellular network. Based on [5], the study in [6] proposed a throughput maximization problem to maximize the rate of an FD-D2D pair which is reusing the spectrum of a cellular user (CU) while fulfilling the data rate constraints of the CU. The works in [6] and [7] derived and analyzed the ergodic sum-rate of an FD-D2D pair who is reusing the bandwidth of a single CU. In [8] a simple power allocation (PA) framework was derived to maximize the throughput of an FD-D2D pair. A closed form PA scheme that maximizes the rate of an FD-D2D network was proposed in [9]. Using the DC programming tool, the authors of [10] proposed a power allocation solution for an FD-D2D network. Giving the tight energy consumption conditions in the upcoming $5 \mathrm{G}$ network the green communication concept is now a crucial criterion in any future network such as the FD-D2D network. Thus, the works in [11], [12] proposed a PA scheme that maximizes the global energy efficiency (GEE) of an FD-D2D based cellular network. Using the Monotonic optimization theory, the authors of [13] derived the optimal PA scheme which maximizes the GEE of an FD-D2D network while fulfilling the QoS of the users.

In all of the mentioned related works, a centralized PA scheme in which the BS needs to know the channel state information of all users at each step is considered. Thus, in practice, the centralized approach will be unfeasible in term of implementation and a decentralized PA solution is needed. In the centralized scenarios, the resources for all the network components are optimized to maximize a system-wide utility function such as sum-rate or GEE. On the contrary, in a decentralized approach, each network node aims to maximize its own utility function by allocating its own resources. Thus, in the decentralized scenario, the network nodes act competitively and in a self-organizing way. Mathematically speaking, the Game theory (GT) framework is the most convenient tool to model and analyze this competitive behavior between the 
network nodes [14]. To the best of the authors knowledge, there is no work used the GT tool to provide a decentralized PA in the context of FD-D2D based cellular network. Fortunately, there is abundant literature on the application of GT for distributed PA in wireless communication networks. For example, the authors of [15], [16] develop a general noncooperative game framework to provide an energy-efficient power control mechanism for a wireless network. The same game was also used in [17] to address the PA problem of a radar network in which multiple radars coexist with a communication system in the same frequency band. Motivated by these works, we propose in this paper a decentralized PA algorithm based on GT analysis for EE maximization in an FD-D2D based cellular network. In particular, we model each user in the network as a rational player who engages in a noncooperative game to allocate the transmission power over its assigned channel. After that, we mathematically proved that this non-cooperative game has a unique equilibrium point that can be achieved through an iterative distributed process. The effectiveness of our distributed algorithm is verified through a numerical simulation which employs the centralized PA scheme proposed in our previous work [13] as a benchmark.

\section{B. Summary of Contributions and paper outline}

The main contributions of this work are as follows:

- Shedding the light on the issue of the distributed implementation of the PA algorithm in an FD-D2D network.

- Providing a distributed PA algorithm based on Game theory for maximizing the EE of an FD-D2D network. To the best of the authors knowledge, none of the existing works provides a decentralized PA scheme of an FDD2D network. To validate the proposed distributed PA algorithm a numerical simulation is conducted which employs the centralized PA scheme proposed in [13] as a benchmark.

- Highlighting the impact of the SI cancellation factor and the D2D proximity distance on the performance of the FD-D2D network.

The rest of this paper is organized as follows. Section II provides the system model and develops the optimization problem. Section III solves the optimization problem using the Game theory analysis and Section IV proposes a distributed iterative algorithm to reach the optimal PA scheme. The simulation results are given in section $\mathrm{V}$, and finally, the conclusion is drawn in section VI.

\section{SYSTEM MODEL AND PROBLEM FORMULATION}

We consider an FD-D2D based cellular network where $M$ half-duplex (HD) CUs coexist with $M$ FD-D2D pairs. Each FD-D2D pair $D 2 D_{j}$ contains two D2D devices $D_{1}^{j}$ and $D_{2}^{j}$ that are in proximity of each other and they are reusing the $j$-th uplink channel assigned to $C U_{j}$. In this work, the uplink (UL) bandwidth reusing scenario is assumed because in this case, the D2D links only impact the BS which is more capable in interference management than the CU. Furthermore, we assume that the CUs are assigned orthogonal UL channels and thus there is no interference between CUs.

Fig. 1 shows an example of this network in which two CUs coexist with two FD-D2D pairs. In this figure, $g_{c, b s}^{j}$ denotes the channel gain between $C U_{j}$ and BS while $g_{d}$ stands for the channel gain between $D_{1}^{j}$ and $D_{2}^{j}$ and between $D_{2}^{j}$ and $D_{1}^{j}$. Moreover, $h_{d 1, b s}^{j}$ and $h_{d 2, b s}^{j}$ respectively denote the interference channel gains from $D_{1}^{j}$ and $D_{2}^{j}$ to BS. $h_{c, d 1}^{j}$ and $h_{c, d 2}^{j}$ respectively represent the interference channel gains from $C U_{j}$ to $D_{1}^{j}$ and $D_{2}^{j}$. All direct/interference channels are assumed to be zero-mean complex Gaussian random variables (i.e., channels are expressing Rayleigh fading) with variance $l_{i j}^{-\alpha}$, where $i \in\{c ; d 1 ; d 2\}, j \in\{b s ; d 1 ; d 2\}, i \neq j, l_{i j}$ is the distance between the nodes $i$ and $j$, and $\alpha$ represents the path-loss exponent. $h_{1,1}^{j}$ and $h_{2,2}^{j}$ denotes the RSI channel at $D_{1}^{j}$ and $D_{2}^{j}$. According to the works in [5], [18], [19] they can be modeled as complex Gaussian random variables with zeromean and variance $\eta p_{d 1}^{j}$ and $\eta p_{d 2}^{j}$ respectively, where $p_{d 1}^{j}$ and $p_{d 2}^{j}$ are respectively the transmission power of $D_{1}^{j}$ and $D_{2}^{j}$, $\eta$ represents the SI cancellation capability of each FD-D2D node.

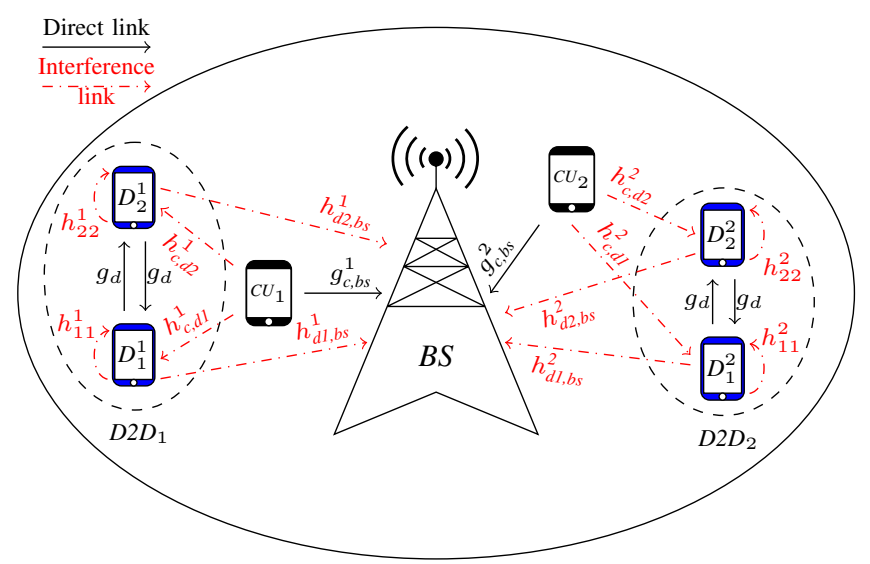

Fig. 1. Full duplex D2D pairs sharing the UL resources of the cellular users.

\section{A. Problem Formulation}

Let $N_{0}$ be the power of the additive white Gaussian noise, and denote by $p_{c}^{j}$ the transmission power of $C U^{j}$. Then, when $D 2 D_{j}$ reuses the spectrum of $C U_{j}$, the signal to interference plus noise ratio (SINR) at the intended receiver of $C U_{j}, D_{1}^{j}$, $D_{2}^{j}$ (i.e., at BS, $D_{2}^{j}$ and $D_{1}^{j}$ ), can be respectively expressed as

$$
\begin{aligned}
\Gamma_{c, r x}^{j} & =\frac{p_{c}^{j} g_{c, b s}^{j}}{I_{-c}+N_{0}}=\frac{p_{c}^{j} g_{c, b s}^{j}}{p_{d 1}^{j} h_{d 1, b s}^{j}+p_{d 2}^{j} h_{d 2, b s}^{j}+N_{0}} \\
\Gamma_{d 1, r x}^{j} & =\frac{p_{d 1}^{j} g_{d}^{j}}{I_{-d 2}+N_{0}}=\frac{p_{d 1}^{j} g_{d}^{j}}{p_{c}^{j} h_{c, d 2}^{j}+\eta p_{d 2}^{j}+N_{0}} \\
\Gamma_{d 2, r x}^{j} & =\frac{p_{d 2}^{j} g_{d}^{j}}{I_{-d 1}+N_{0}}=\frac{p_{d 2}^{j} g_{d}^{j}}{p_{c}^{j} h_{c, d 1}^{j}+\eta p_{d 1}^{j}+N_{0}}
\end{aligned}
$$

where $j \in\{1,2, \ldots, M\}$, and $I_{-k}$ stands for the interference on the intended receiver of the $k$-th transmitter with $k \in\{c, d 1, d 2\}$. 
Now, using Shannon theorem, the rate (in bits/sec/Hz) of the $j$-th $D 2 D$ links and the $j$-th cellular link are given by:

$$
R_{k}^{j}\left(p_{k}^{j}, \mathbf{p}_{-k}^{j}\right)=\log _{2}\left(1+\Gamma_{k, \mathrm{rx}}^{j}\right), k \in\{c, d 1, d 2\}, j \in\{1, \ldots, M\}
$$

with $p_{k}^{j}$ being the transmission power of node $k$ on the $j$-th channel and $\mathbf{p}_{-k}^{j}$ being the interference power vector at node $k$ 's intended receiver on the $j$-th channel containing all powers except user $k$ 's.

The energy efficiency (EE) of the above links measured in (bit/Joule/Hz) is defined as the energy cost of the communication link, and it is expressed as:

$$
E E_{k}^{j}\left(p_{k}^{j}, \mathbf{p}_{-k}^{j}\right)=\frac{R_{k}^{j}\left(p_{k}^{j}, \mathbf{p}_{-k}^{j}\right)}{p_{k}^{j}+P_{c i r}}, k \in\{c, d 1, d 2\}, j \in\{1, \ldots, M\}
$$

where $P_{\text {cir }}$ is the wasted circuit power during the transmission at any mobile device.

Considering the energy cost of the D2D and cellular links and the QoS constraints of the users, we aim in this work to propose an energy-efficient distributed PA algorithm for an FD-D2D network. From a mathematical prospective, this can be done by jointly solving the following $\mathrm{EE}$ maximization problems:

$$
\begin{gathered}
\arg \max _{p_{k}^{j} \in \mathcal{P}_{k}} E E_{k}^{j}\left(p_{k}^{j}, \mathbf{p}_{-k}^{j}\right), \forall k \in\{c, d 1, d 2\}, j \in\{1, \ldots, M\} \text { (6) } \\
\mathcal{P}_{k}^{j}=\left\{p_{k}^{j} \in \mathbb{R}_{+}: p_{k}^{j} \leq p_{\max }^{k}, \Gamma_{k, r x}^{j} \geq \gamma_{\min }^{k, j}\right\}
\end{gathered}
$$

with $\mathcal{P}_{k}^{j}$, being the feasible set of the $k$ th user transmitting on the $j$-th channel. $P_{\max }^{k}$ is the maximum transmission power of a transmitter $k$, and $\gamma_{\text {min }}^{k, j}$ is the minimum required SINR level to achieve the minimum data rate of the $k$ th user who is operating on the $j$-th channel. Solving these coupled maximization problems is the target of the next section.

\section{GAMe Theory BASEd Power Allocation ALGORITHM}

The aim of this section is to reformulate the optimization problems defined in (6) as game using the game theory, and show that this game admits a unique solution which can be obtained through an iterative process. For ease of notation, and since the couples $\left(D 2 D_{j}, C U_{j}\right)$ with $j=\{1,2, \ldots, M\}$ are independent of each others, in the sequel we remove the $j$-th superscript from our analysis, i.e., we solve the optimization problem of a generic $D 2 D$ pair that is reusing the uplink resource of an $C U$.

\section{A. Game-Theoretical Problem Formulation}

The coupled interference among the D2D and cellular links brings interactions between the D2D users and CUs that look to maximize their EE while satisfying their own QoS constraints (6). Such scenario can be analyzed using the non-cooperative game theory. In particular, the maximization problems in (6) can be formulated as the non-cooperative game in normal form:

$$
\mathcal{G} \triangleq\left\{\mathcal{K},\left\{\mathcal{P}_{k}\right\}_{k \in \mathcal{K}},\left\{E E_{k}\left(p_{k}, \mathbf{p}_{-k}\right)\right\}_{k \in \mathcal{K}}\right\}
$$

where:

- $\mathcal{K}=\left\{c, d_{1}, d_{2}\right\}$ is the set of players. Here, the elements of $\mathcal{K}$ are respectively indicating the $C U, D_{1}$, and $D_{2}$.

- $\mathcal{P}_{k}$ is the strategy set of player $k$.

- $E E_{k}\left(p_{k}, \mathbf{p}_{-k}\right)$ is player $k$ 's utility function.

Moreover, the optimization problems in (6) are known as the best-response dynamics (BRD) of the game $\mathcal{G}$, and the solution of the $k$-th optimization problem in (6) defines the best-response (BR) of the $k$-th player to the remaining players' actions $\mathbf{p}_{-k}$. Hence, we define the $k$ th player's BR $\mathcal{B}_{k}\left(\mathbf{p}_{-k}\right)$ as

$$
\mathcal{B}_{k}\left(\mathbf{p}_{-k}\right) \triangleq \arg \max _{p_{k} \in \mathcal{P}_{-k}} E E_{k}\left(p_{k}, \mathbf{p}_{-k}\right) .
$$

Any steady point of the BRD represents a solution of $\mathcal{G}$ and it is called a Nash equilibrium (NE) point. generally speaking, a non-cooperative game may have zero, one, or multiple NE points. Further, in the case of the existence of an equilibrium point, the convergence of the BRD is not guaranteed. Accordingly, the main concerns when analyzing a non-cooperative game are to prove the existence and uniqueness of an NE and to show the convergence of BRD towards this unique NE.

\section{B. Existence of an Equilibrium}

The aim of this sub-section is to prove the existence of an NE solution for the power allocation game $\mathcal{G}$. To that end, first, we present the following two Lemmas:

Lemma 1. The energy efficiency function $E E_{k}$ is strictly quasiconvex for $p_{k} \in \mathbb{R}_{+}$, and the rate function $R_{k}$ is strictly convex for $p_{k} \in \mathbb{R}_{+}$.

Proof: First, let us show the convexity of $R_{k}\left(\Gamma_{k, \mathrm{rx}}\left(p_{k}\right)\right)$. Applying the chain rule, the second-order derivative of $R_{k}$ can be expressed as

$$
\frac{\partial^{2} R_{k}}{\partial^{2} p_{k}}=\underbrace{\frac{\partial R_{k}}{\partial \Gamma_{k, \mathrm{rx}}} \frac{\partial^{2} \Gamma_{k, \mathrm{rx}}}{\partial p_{k}^{2}}}_{A}+\underbrace{\frac{\partial^{2} R_{k}}{\partial \Gamma_{k, \mathrm{rx}}^{2}}}_{B} \underbrace{\left(\frac{\partial \Gamma_{k, \mathrm{rx}}}{\partial p_{k}}\right)^{2}}_{C}
$$

Now, to prove the convexity of $E E_{k}$ we must show that (10) is always negative. It is easily found that $\frac{\partial^{2} \Gamma_{k, \mathrm{rx}}}{\partial p_{k}^{2}}=0$, thus $A$ is equal to zero. Moreover, we observe that $C$ is always greater than zero. Hence, the convexity of $E E_{k}$ only depends on $B$. Computing $B$ shows that it is always negative and it is equal to $\frac{-1}{\left(1+\Gamma_{k, \mathrm{rx}}\right)^{2} \ln (2)}$. Hence, the convexity of $R$ is proved. Then, $E E_{k}$ is given by the ratio between a strictly concave function $\left(R_{k}\right)$ and a linear function, which results in a strictly pseudo-concave function [14]. Moreover, any strictly pseudo convex function is also quasi-concave [14], thus Lemma 1 is proved.

\section{Lemma 2. If}

$\left\{\begin{array}{l}p_{\max }^{c} \geq \frac{\gamma_{\min }^{c}}{g_{c, b s}}\left(p_{d_{1}} h_{d 1, b s}+p_{d_{2}} h_{d 2, b s}+\sigma_{N}^{2}\right) \triangleq P_{\min }^{c}\left(\boldsymbol{p}_{-c}\right), \\ p_{\max }^{d 2} \geq \frac{\gamma_{\min }^{d 1}}{g_{d}}\left(P_{c} g_{c, d 1}+\eta P_{d 1}+\sigma_{N}^{2}\right) \triangleq P_{\min }^{d 2}\left(\boldsymbol{p}_{-d 2}\right),(11 \\ P_{\max }^{d 1} \geq \frac{\gamma_{\min }^{d 2}}{g_{d}}\left(P_{c} g_{c, d 2}+\eta P_{d 2}+\sigma_{N}^{2}\right) \triangleq P_{\min }^{d 1}\left(\boldsymbol{p}_{-d 1}\right),\end{array}\right.$ 
then the BR of the players are:

$$
\mathcal{B}_{k}\left(\boldsymbol{p}_{-k}\right)=\min \left\{p_{\max }^{k}, \max \left\{p_{k}^{\star}, p_{\min }^{k}\right\}\right\} \forall k \in \mathcal{K} .
$$

wherein

$$
p_{k}^{\star}=\arg \max _{p_{k}} E E_{k}\left(p_{k}, \boldsymbol{p}_{-k}\right) \text { s.t } p_{k} \in \mathbb{R}_{+} .
$$

Proof: The first part of Lemma 2 can be obtained by using the fact that $p_{k} \leq p_{\max }^{k}$ for all $k \in \mathcal{K}$ and reformulating the QoS requirements $\gamma_{k} \geq \gamma_{\min }^{k} \forall k \in \mathcal{K}$ as

$p_{c} \geq \frac{\gamma_{\min }^{c}\left(I_{-c}+N_{0}\right)}{g_{c, b s}}, p_{d_{2}} \geq \frac{\gamma_{\min }^{d 1}\left(I_{-d_{2}}+N_{0}\right)}{g_{d}}, p_{d_{1}} \geq \frac{\gamma_{\min }^{d 2}\left(I_{-d_{1}}+N_{0}\right)}{g_{d}}$.

Hence if (11)-(11b) hold, there is always a power $p_{k} \in$ $\left[0, p_{\text {max }}^{k}\right]$ such that the QoS constraint $\Gamma_{k, \mathrm{rx}} \geq \gamma_{\min }^{k}$ is satisfied for all $k \in \mathcal{K}$.

To prove the second part of Lemma 2, first, we note that $E E_{k}$ is a concave function with $p_{k}$ (See Lemma 1). Thus, it has a unique optimal point $p_{k}^{\star} \in \mathbb{R}_{+}$. Hence, $E E_{k}$ is increasing for $p_{k} \leq p_{k}^{\star}$ and decreasing for $p_{k} \geq p_{k}^{\star}$. This means that if $p_{k}^{\star}>$ $p_{\max }^{k}$, then $p_{\max }^{k}$ is the maximizer of $E E_{k}$. Next, by considering the QoS and maximum power constraints, and applying (11)(11b), we obtain (12).

Now, we show that in our context the game $\mathcal{G}$ always admits an NE assuming that (11)-(11a) are satisfied.

The author of [20] showed that the existence of an NE is guaranteed under the following assumptions:

1) The feasible action sets $\mathcal{P}_{k}$ of the players are nonempty, closed, and contained in some compact set $\mathcal{C}_{k}$ for all $\mathbf{p}_{-k} \in \mathcal{P}_{-k} \equiv \prod_{l \neq k} \mathcal{P}_{l}$.

2) The sets $\mathcal{P}_{k}$ vary continuously with $\mathbf{p}_{-k}$ (in the sense that the graph of the set-valued correspondence $\mathbf{p}_{-k} \mapsto \mathcal{P}_{k}$ is closed).

3) The utility function $E E_{k}\left(p_{k}, \mathbf{p}_{-k}\right)$ of each user is quasi concave in $\mathbf{p}_{-k}$ for all $\mathbf{p}_{-k} \in \mathcal{P}_{k}$.

In our framework, when (11)-(11b) hold true, then the feasible sets $\mathcal{P}_{k}$ are non-empty, convex (since based on Lemma $1 R_{k}=$ $\log _{2}\left(1+\Gamma_{k, \mathrm{rx}}\right)$ is convex in $\left.p_{k}\right)$, closed and bounded for every $\mathbf{p}_{-k}$. Moreover, all the sets $\mathcal{P}_{k}$ vary continuously with $\mathbf{p}_{-k}$ because the QoS requirements $\Gamma_{k} \geq \gamma_{\min }^{k}$ is continuous in $\mathbf{p}_{-k}$ for all $k \in\{c, d 1, d 2\}$. Besides, and based on Lemma 1 , the utility function $E E_{k}$ is quasi-concave function. Thus, in our scenario, all the above three conditions are satisfied and the power allocation game $\mathcal{G}$ admits at least one NE point. The following section shows that $\mathcal{G}$ has a unique NE point, and that BRD always converges to such point.

\section{Analysis of the equilibria}

According to [21] a non-cooperative game has a unique $\mathrm{NE}$ which can be acieved by iteratively solving the BR of the players if: 1) the game has a non-empty set of NE points and 2) the BR function is a standard function. In the previous section we have already shown that $\mathcal{G}$ has at least one NE point. Thus, it only remains to prove that the $\mathrm{BR}$ of the players defined in
(12) are standard functions ${ }^{1}$. In [14] it is proved that $p_{k}^{\star}$ is a standard function. On the other hand, since $N_{0}$ is always positive, $P_{\min }^{k} \forall k \in \mathcal{K}$ in (11)-(11a) is non-negative function. Moreover, it is also monotonic function because it is increasing in all $\left\{p_{j}\right\}_{j \neq k}$. Now, to prove the scalability feature of $P_{\mathrm{min}}^{k}$, take any scaling factor $\beta>1$, then we will have

$$
\begin{gathered}
P_{\min }^{c}\left(\beta \mathbf{p}_{-c}\right)=\beta \gamma_{\min }^{c} \frac{I_{c}+\frac{N_{0}}{\beta}}{g_{c, b s}}<\beta \frac{I_{c}+N_{0}}{g_{c, b s}}=\beta P_{\min }^{c}\left(\mathbf{p}_{-c}\right),(15) \\
P_{\min }^{d 2}\left(\beta \mathbf{p}_{-d 2}\right)=\beta \gamma_{\min }^{d 2} \frac{I_{d 2}+\frac{N_{0}}{\beta}}{g_{d}}<\beta \frac{I_{d 2}+N_{0}}{g_{d}}=\beta P_{\min }^{d 2}\left(\mathbf{p}_{-d 2}\right), \\
P_{\min }^{d 1}\left(\beta \mathbf{p}_{-d 1}\right)=\beta \gamma_{\min }^{d 2} \frac{I_{d 2}+\frac{N_{0}}{\beta}}{g_{d}}<\beta \frac{I_{d 1}+N_{0}}{g_{d}}=\beta P_{\min }^{d 1}\left(\mathbf{p}_{-d 1}\right) .
\end{gathered}
$$

At this stage, we have shown that both $P_{\min }^{k}$ and $p_{k}^{\star}$ are standard functions. Now, observe that $P_{\max }^{k}$ does not depend on $\mathbf{p}_{k}$ for all $k \in \mathcal{K}$, it follows that the best responses defined in (12) are standard functions because both $\max ($.$) and \min ($. are increasing functions. As a result, the power allocation game $\mathcal{G}$ admits a unique solution and and its BRC converges to this unique point. The next section provides a distributed algorithm that can solve $\mathcal{G}$.

\section{DistribUted POWER ALLOCATION ALGORITHM}

In this section, we aim at providing a distributed power allocation algorithm that converges to the unique NE point of the game $\mathcal{G}$. To do so, we need to derive the point $p_{k}^{\star}$ defined in (13). For ease of notation, we denote by $\mu_{k} \triangleq I_{-k}+N_{0}$ the equivalent interference gain on the $k$-th link, and call $\gamma_{\text {max }}^{c} \triangleq$ $g_{c, b s}, \gamma_{\max }^{d 1} \triangleq g_{d}, \gamma_{\max }^{d 2} \triangleq g_{d}$ the maximum SINRs values that can be obtained at the cellular link and the D2D links respectively when these nodes does not receiving interference and the thermal noise is negligible. Using these definitions the SINRs defined in (1)-(3) can be rewritten as

$$
\Gamma_{k, \mathrm{rx}}=\frac{\gamma_{\max }^{k}}{\mu_{k}} p_{k} \forall k \in \mathcal{K} .
$$

Now, the solution of (13), $p_{k}^{\star}$, is given in the following Lemma.

Lemma 3. For any given $\boldsymbol{p}_{-k}$, $p_{k}^{\star}$ is given by

$$
p_{k}^{\star}=\Phi_{k}\left(\lambda_{k}^{\star}\right)=\left[\frac{1}{\lambda_{k}^{\star}}-\frac{\mu_{k}}{\gamma_{\text {max }}^{k}}\right]^{+}
$$

with $\lambda^{\star}$ being the root of the following function:

$$
F\left(\lambda_{k}\right)=\max _{p_{k} \in \mathbb{R}_{+}} \log _{2}\left(1+\frac{\gamma_{\max }^{k}}{\mu_{k}} p_{k}\right)-\lambda_{k}\left(p_{k}+P_{c i r}\right) .
$$

Proof: Observe that $E E_{k}$ is a ratio of two functions. Hence, it belongs to the class of fractional programming theory. Consequently, the solution of (13) can be found by means of Dinkelbach's algorithm [22]. By means of the latter, solving the EE maximization problem in (13) is equivalent to

\footnotetext{
${ }^{1}$ A function $f(\mathbf{x})$ is standard if it fulfills the properties of $i$ ) Positivity: $f(\mathbf{x}) \geq 0$ for all $\mathbf{x} \geq 0$; ii) Monotonicity: $f\left(\mathbf{x}_{1}\right) \geq f\left(\mathbf{x}_{2}\right) \forall \mathbf{x}_{1} \succeq \mathbf{x}_{2} ;$ iii $)$ Scalability: $f(\beta \mathbf{x})<\beta f(\mathbf{x})$ for all $\mathbf{x} \geq 0, \beta>1$.
} 
finding the solution $\lambda_{k}^{\star}$ of (18). Now, taking the derivative of (18) w.r.t $p_{k}$ and setting it to zero results in:

$$
\frac{1}{1+\Gamma_{k, \mathrm{rx}}} \frac{\partial \Gamma_{k, \mathrm{rx}}}{\partial p_{k}}-\lambda_{k}=0 .
$$

Using (16), $\frac{\partial \Gamma_{k, \mathrm{rx}}}{\partial p_{k}}=\frac{\gamma_{\max }^{k}}{\mu_{k}}$ and taking into account that $p_{k}^{\star} \geq 0$ eventually yield to (17).

Now let $p_{k}[i]$ be the $k$-th player's transmit power at the $i$-th iteration step. Based on the results of Section II and Lemma 3, there is an algorithm that converges to the unique NE of $\mathcal{G}$ and it operates by iteratively updating the players' BR

$$
p_{k}[i+1]=\min \left\{P_{\max }^{k}, \max \left\{p_{k}^{\star}[i], P_{\min }^{k}[i]\right\}\right\}
$$

where $P_{\min }^{k}[i]$ is calculated using (16) as follows

$$
P_{\min }^{k}[i]=\frac{\gamma_{\min }^{k}}{\gamma_{\max }^{k}} \mu_{k}[i] .
$$

Algorithm 1 presents the pseudo-code of this iterative process. From (17) and (18) it is clear that the calculation of $p_{k}[i+1]$ in (20) needs only information about $\mu_{k}[i]$ because $\gamma_{\max }^{k}, \gamma_{\in}^{k}$, and $P \max ^{k}$ are locally available at each receiver. Now, to earn this info we reformulate $\mu_{k}[i]$ using (16) as

$$
\mu_{k}[i]=\frac{\gamma_{\max }^{k}}{\Gamma_{k, \mathrm{rx}}[i]} p_{k}[i]
$$

where $\Gamma_{k, \mathrm{rx}}[i]$ is the SINR of the $k$-th node's intended receiver at the $i$-th iteration, and $p_{k}[i]$ is the transmit power of the $k$-th receiver's intended transmitter at the $i$-th iteration. Inspecting (22) shows that $\mu_{k}[i]$ can be easily obtained when each transmitter knows the SINR value at its intended receiver $\Gamma_{k, \mathrm{rx}}[i]$. This is because each node knows the value of its transmit power $p_{k}[i]$ and its maximum achievable SINR value $\gamma_{\max }^{k}$. Note that, the intended receiver of a transmitter $k$ can easily estimate its SINR and return it to the k-th transmitter using a feedback downlink channel. Thus, the BRD process reported in Algorithm 1 can be executed in a fully distributed manner.

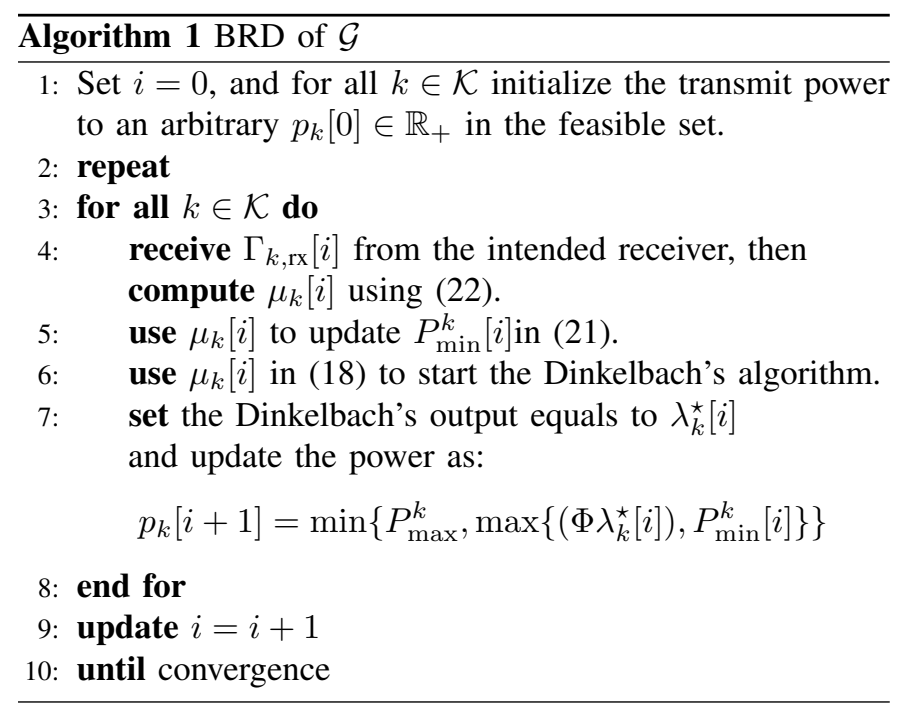

\section{Simulation RESUlts}

In this section, numerical simulations under different operating conditions are used to analyze the performance of the proposed distributed PA (Algorithm 1). Moreover, since none of the previous work address the distributed implementation of PA in the context of FD-D2D network, we compare Algorithm 1 with two power allocation strategies: i) the centralized power allocation algorithm in [13] which aims to maximize the global energy efficiency of the FD-D2D network (GEE) defined as $G E E=\frac{\sum_{k \in \mathcal{K}} R_{k}}{\sum_{k \in \mathcal{K}} p_{k}+3 P_{\text {cir }}}$, ii) the fixed power allocation strategy in which each user transmits at its maximum allowed transmission power $P_{\max }^{k}$.

Besides, three metrics are used to assess the performance:

(i) the D2D rate gain brought by the accessed FD-D2D pairs defined as $R_{\text {gain }} \triangleq \frac{\sum_{k \in \mathcal{K}} R_{k}-R_{c}^{\max }}{R_{c}^{\max }} \times 100$, with $R_{c}^{\max }=\log _{2}\left(1+\frac{g_{c, b s}}{N_{0}} P_{\max }^{c}\right)$ being the maximum achievable cellular link throughput when the FD-D2D pairs do not exist,

(ii) the rate of the FD-D2D pair $R_{d 2 d}$ defined as $R_{d 2 d}=R_{d 1}+R_{d 2}$

(iii) and the GEE of the accessed couples (D2D, CU).

\section{A. Simulation setup}

We consider a single cell network with radius $R=500 \mathrm{~m}$ in which $M$ FD-D2D pairs coexist with $M$ CUs. The CUs are equally sharing the uplink bandwidth and they are uniformly distributed in the cell. Moreover, each $D 2 D_{j}$ is sharing the same resource of $C U_{j}$ and it is uniformly distributed within a randomly located cluster with radius $r$. Throughout our simulation, we consider 500 realizations each with $M=15$ CUs and $M=15 \mathrm{D} 2 \mathrm{D}$ pairs and the results are averaged over the $M$ couples $\left(D 2 D_{j}, C U_{j}\right)$ and the 500 realizations. Besides, in our simulation, the convergence of Algorithm 1 is declared when the relative squared error between the achieved power vector $\mathbf{P}=\left[p_{c}, p_{d 1}, P_{d 2}\right]$ values between two successive iterations is not larger than $10^{-6}$. All the simulation parameters are summarized in Table I.

TABLE I

SIMULATION PARAMETERS

\begin{tabular}{|l|l|}
\hline Cell radius $(\mathrm{R})$ & $500 \mathrm{~m}$ \\
\hline D2D cluster radius(r) & $10,20,40,80(\mathrm{~m})$ \\
\hline Number of CUs and D2D pairs $(\mathrm{M})$ & 15 \\
\hline Noise power $\left(N_{0}\right)$ & $-114 \mathrm{dBm}$ \\
\hline Path-loss exponent $(\alpha)$ & 4 \\
\hline Maximum power of CUE and D2D users & $24 \mathrm{dBm}$ \\
\hline SINR requirement for CUs and D2D $\left(\gamma_{\min }^{k}\right)$ & $\begin{array}{l}\text { uniform distributed in } \\
{[0,25] \mathrm{dB}}\end{array}$ \\
\hline SI cancellation factor $(\eta)$ & $-100,-90, \ldots,-50 \mathrm{~dB}$ \\
\hline Multiple-path fading & $\begin{array}{l}\text { Exponential distribu- } \\
\text { tion with } l_{i j}^{-\alpha} \text { mean }\end{array}$ \\
\hline Convergence criterion of Algorithm 1 & $\frac{\|\mathbf{P}[i]-\mathbf{P}[i-1]\| \|^{2}}{\|\mathbf{P}[i]\| \|^{2}} \leq 10^{-6}$ \\
\hline
\end{tabular}

\section{B. Numerical assessment}

Considering the above simulation setup, we show in Fig.2 the behavior of FD-D2D rate gain with respect to (w.r.t.) the 
self-interference cancellation factor $\eta$ and the D2D cluster radius $r$. As it can be seen, $R_{\text {gain }}$ increases as $\eta$ decreases. This is because at low $\eta$ the self-interference decreases which reflects in lower interference and higher rate. Moreover, the FD-D2D rate gain decreases as $r$ increases because when $r$ increases more power is needed to meet the QoS which results in higher interference and lower rate. Thus, to achieve the highest possible rate gain of an FD-D2D network, the D2D users must be close to each other and the SI cancellation capability must be very low.

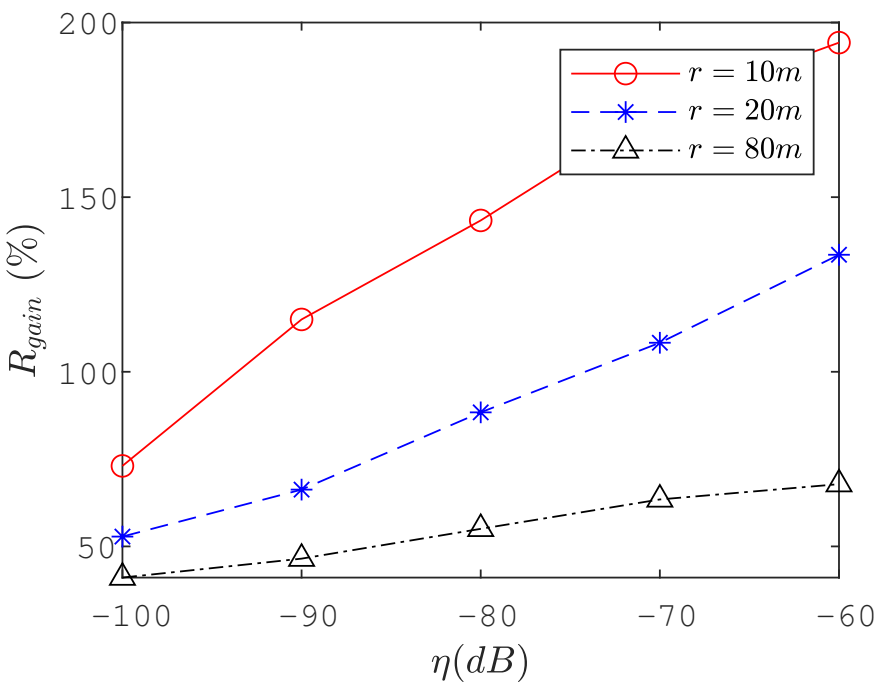

Fig. 2. The rate gain behavior of the proposed distributed PA w.r.t. the selfinterference cancellation factor $\eta$ and D2D proximity distance $r$.

Focusing on the case of small D2D distance $(r=10 \mathrm{~m})$, Fig. 3 compares the GEE of the links for the three power allocation strategies. As expected the centralized PA algorithm proposed in [12] outperforms our proposed distributed algorithm, as well as the full-power transmission algorithm, in terms of global energy efficiency. This is because the centralized algorithm originally created to maximize the GEE of an FD-D2D network, while our proposed distributed algorithm aims to find an equilibrium point when each users seeks to selfishly maximize its own EE. Yet, the proposed distributed algorithm reaches $84 \%$ of the optimal GEE point achieved by the centralized solution at $\eta=-100 d B$. Notice that the proposed distributed power allocation requires much less feedback overhead comparing to the centralized approach which makes it more attractive for real implementation.

Now, to have a complete picture about the performance of our proposed algorithm, we set $r=10 \mathrm{~m}$ and we show in Fig. 4 the achieved D2D rate by the three PA strategies. As it can be seen, our proposed algorithm achieves higher D2D rate comparing to both the centralized solution and the full power transmission algorithm. This is because our proposed algorithm consumes more energy comparing to the centralized solution (See Fig. 3). Since the aim of an FD-D2D network is to offload as much data as possible from the cellular network, the proposed distributed algorithm seems to be much more attractive for the FD-D2D network designers. Moreover,

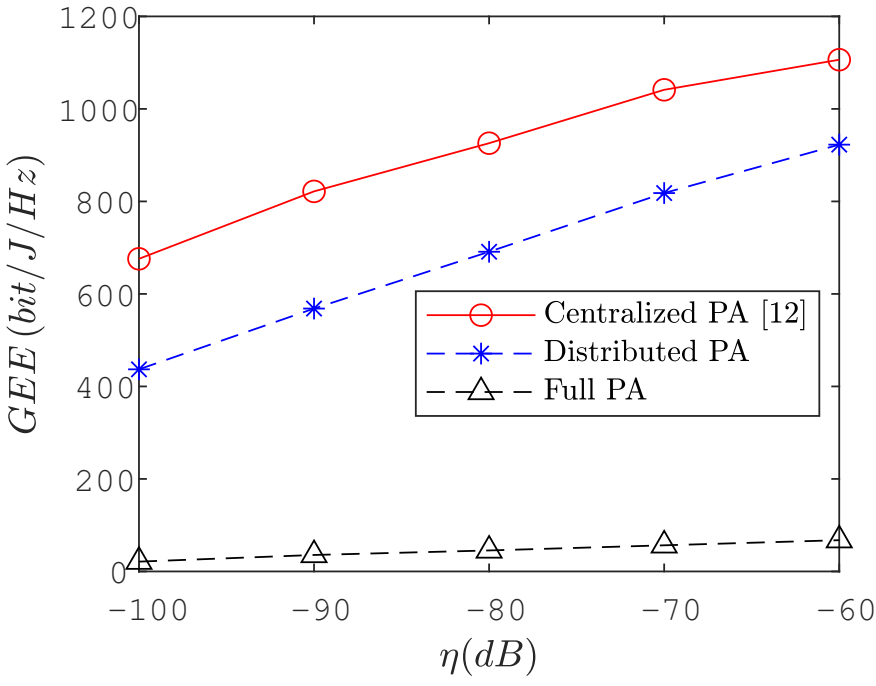

Fig. 3. The GEE behavior w.r.t. the self-interference cancellation factor $\eta$ when allocating power according to: 1) the centralized PA algorithm proposed in $[12], 2)$ the proposed distributed PA algorithm, and 3) the full-power transmission strategy.

from both Fig.4 and Fig.3, we can see that the full-power transmission algorithm is the worst strategy in terms of both $\mathrm{EE}$ and rate. This is because, transmission at the maximum power when users are in low proximity distances yields to high interference and low rate.

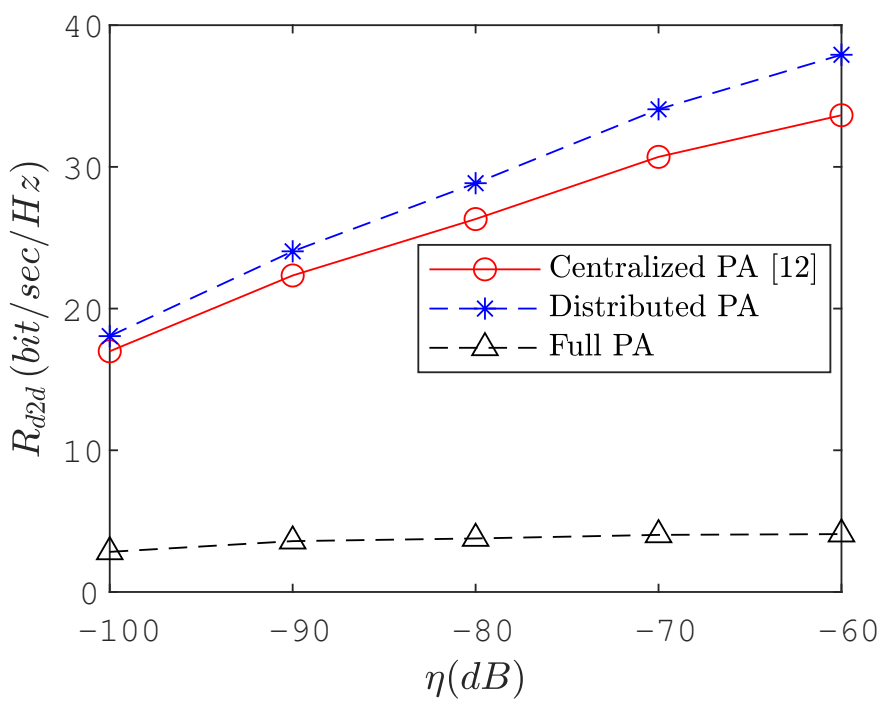

Fig. 4. The achieved D2D rate when allocating power according to: 1) the centralized PA algorithm proposed in [12], 2) the proposed distributed PA algorithm, and 3) the full-power transmission strategy.

Finally, to show the superiority of our proposed algorithm in terms of complexity, we present in Table.V-B the average execution time of the proposed distributed algorithm and the centralized algorithm. As it can be seen, the distributed algorithm required much more less time to achieve the equilibrium. This is because the centralized approach needs to solve a convex problem at each iteration using the well known interior point method. 
TABLE II

THE AVERAGE EXECUTION TIME OF THE PROPOSED DISTRIBUTED ALGORITHM AND THE CENTRALIZED ALGORITHM.

\begin{tabular}{c|cc|cc}
\hline \multirow{2}{*}{$\eta(d B)$} & \multicolumn{2}{|c|}{$r=10(\mathrm{~m})$} & \multicolumn{2}{c}{$r=20(\mathrm{~m})$} \\
& Centralized & Distributed & Centralized & Distributed \\
\hline-60 & $1429.1 \mathrm{~ms}$ & $0.013 \mathrm{~ms}$ & $1139.2 \mathrm{~ms}$ & $0.09 \mathrm{~ms}$ \\
-70 & $1228.1 \mathrm{~ms}$ & $0.01 \mathrm{~ms}$ & $1323.8 \mathrm{~ms}$ & $0.01 \mathrm{~ms}$ \\
-80 & $943.1 \mathrm{~ms}$ & $0.01 \mathrm{~ms}$ & $940.5 \mathrm{~ms}$ & $0.01 \mathrm{~ms}$ \\
-90 & $656.4 \mathrm{~ms}$ & $0.01 \mathrm{~ms}$ & $747.5 \mathrm{~ms}$ & $0.01 \mathrm{~ms}$ \\
-100 & $442 \mathrm{~ms}$ & $0.01 \mathrm{~ms}$ & $513.2 \mathrm{~ms}$ & $0.01 \mathrm{~ms}$ \\
\hline
\end{tabular}

\section{CONCLUSION}

In this paper we address the power allocation problem of an FD-D2D based cellular network. In particular, we propose a distributed PA algorithm for such kind of network by using Game theory analysis. First, we model the interactions between the CUs and the D2D users as a non-cooperative game $\mathcal{G}$. Next, we show that $\mathcal{G}$ admits a unique Nash equilibrium point which can be achieved through an iterative algorithm. After that we show that this iterative algorithm can be implemented in a fully distributed manner. Simulation results verify the effectiveness of our proposed distributed power allocation algorithm and show that the self-interference capability and the proximity distance of the D2D devices highly affect the performance of the FD-D2D network. As a future work, we will address the issue of designing a distributed joint channel assignment and power allocation algorithm.

\section{ACKNOWLEDGEMENT}

This work was partially funded through the Lebanese University (LU) research center. A part of this work was also carried out thanks to the fund from the French National Research Agency (ANR) project WONG5 with grant agreement code: ANR-15-CE25-0005603.

\section{REFERENCES}

[1] V. Cisco Mobile, "Cisco visual networking index: Global mobile data traffic forecast update, 2016-2021 white paper," 2017.

[2] K. Doppler, M. Rinne, C. Wijting, C. B. Ribeiro, and K. Hugl, "Deviceto-device communication as an underlay to lte-advanced networks," IEEE Communications Magazine, vol. 47, no. 12, 2009.

[3] A. Sabharwal, P. Schniter, D. Guo, D. W. Bliss, S. Rangarajan, and R. Wichman, "In-band full-duplex wireless: Challenges and opportunities," IEEE Journal on Selected Areas in Communications, vol. 32, no. 9, pp. 1637-1652, 2014

[4] V. Tapio, "System scenarios and technical requirements for full-duplex concept," DUPLO, Project Deliverable, 2013.

[5] W. Cheng, X. Zhang, and H. Zhang, "Optimal power allocation for fullduplex d2d communications over wireless cellular networks," in 2014 IEEE Global Communications Conference, Dec 2014, pp. 4764-4769.

[6] K. T. Hemachandra, N. Rajatheva, and M. Latva-aho, "Sum-rate analysis for full-duplex underlay device-to-device networks," in 2014 IEEE Wireless Communications and Networking Conference (WCNC), April 2014, pp. 514-519.

[7] H. Chour, F.Bader, Y. Nasser, and O. Bazzi, "Full-duplex or half-duplex d2d mode? closed form expression of the optimal power allocation," in 25th International Conference on Telecommunication, 2018.

[8] H. Chour, O. Bazzi, F. Bader, and Y. Nasser, "Analytical Framework for Joint Mode Selection and Power Allocation for Full Duplex D2D Network," in IEEE Wireless Communications and Networking Conference (WCNC'2019), marrakech, Morocco, Apr. 2019. [Online]. Available: https://hal.archives-ouvertes.fr/hal-02002842
[9] H. Chour, F. Bader, Y. Nasser, and O. Bazzi, "GALEN: A Geometric Framework for Global Optimal Power Allocation in a Full Duplex D2D Network," in IEEE Wireless Communications and Networking Conference (WCNC'2019), Marrakech, Morocco, Apr. 2019. [Online]. Available: https://hal.archives-ouvertes.fr/hal-02002848

[10] S. Li, Q. Ni, Y. Sun, and G. Min, "Resource allocation for weighted sum-rate maximization in multi-user full-duplex device-to-device communications: Approaches for perfect and statistical csis," IEEE Access, vol. 5, pp. 27 229-27241, 2017.

[11] J. Ding, L. Jiang, and C. He, "Energy-efficient power control for underlaying full-duplex device-to-device communications," in Proc. Asia Modelling Symp. (AMS), Dec. 2017, pp. 155-160.

[12] R. Tang, J. Zhao, H. Qu, and Z. Zhang, "Energy-efficient resource allocation for $5 \mathrm{~g}$ full-duplex enabled device-to-device communication," in Proc. IEEE Globecom Workshops (GC Wkshps), Dec. 2016, pp. 1-7.

[13] H. Chour, E. A. Jorswieck, F. Bader, Y. Nasser, and O. Bazzi, "Global optimal resource allocation for efficient fd-d2d enabled cellular network," IEEE Access, vol. 7, pp. 59690-59707, 2019.

[14] A. Zappone, E. Jorswieck et al., "Energy efficiency in wireless networks via fractional programming theory," Foundations and Trends $\AA$ in Communications and Information Theory, vol. 11, no. 3-4, pp. 185-396, 2015.

[15] A. Zappone, L. Sanguinetti, G. Bacci, E. Jorswieck, and M. Debbah, "Energy-efficient power control: A look at 5g wireless technologies," IEEE Transactions on Signal Processing, vol. 64, no. 7, pp. 1668-1683, April 2016.

[16] G. Bacci, E. V. Belmega, P. Mertikopoulos, and L. Sanguinetti, "Energyaware competitive power allocation for heterogeneous networks under qos constraints," IEEE Transactions on Wireless Communications, vol. 14, no. 9, pp. 4728-4742, Sep. 2015.

[17] C. Shi, F. Wang, M. Sellathurai, and J. Zhou, "Non-cooperative game theoretic power allocation strategy for distributed multiple-radar architecture in a spectrum sharing environment," IEEE Access, vol. 6, pp. 17 787-17 800, 2018.

[18] B. Zuo, L. Jiang, C. He, and Z. Lian, "Power allocation optimization for full-duplex $\mathrm{d} 2 \mathrm{~d}$ communications underlaying cellular networks," in 2016 International Conference on Networking and Network Applications (NaNA). IEEE, 2016, pp. 103-108.

[19] T. Riihonen, S. Werner, and R. Wichman, "Mitigation of loopback selfinterference in full-duplex mimo relays," IEEE Transactions on Signal Processing, vol. 59, no. 12, pp. 5983-5993, 2011.

[20] G. Debreu, "A social equilibrium existence theorem," Proceedings of the National Academy of Sciences, vol. 38, no. 10, pp. 886-893, 1952.

[21] R. D. Yates et al., "A framework for uplink power control in cellular radio systems," IEEE Journal on selected areas in communications, vol. 13, no. 7, pp. 1341-1347, 1995.

[22] J.-P. Crouzeix and J. A. Ferland, "Algorithms for generalized fractional programming," Mathematical Programming-Springer, vol. 52, pp. 191207, 1991. 